\title{
Pretreatment with the ALDH2 activator Alda-1 protects rat livers from ischemia/reperfusion injury by inducing autophagy
}

\author{
ZHONGZHONG LIU $^{1 *}$, SHAOJUN YE $^{1 *}$, XIANG ZHONG ${ }^{2}$, WEI WANG ${ }^{1}$, CHIN-HUI LAI $^{1}$, \\ WANG YANG $^{1}$, PENGPENG YUE ${ }^{1}$, JUN LUO ${ }^{1}$, XIAOYING HUANG $^{1}$, ZIBIAO ZHONG ${ }^{1}$, \\ YAN XIONG $^{1}$, XIAOLI FAN ${ }^{1}$, LING LI $^{1}$, YANFENG WANG ${ }^{1}$ and QIFA YE ${ }^{1,3}$ \\ ${ }^{1}$ Zhongnan Hospital of Wuhan University, Institute of Hepatobiliary Diseases of Wuhan University, Transplant Center of \\ Wuhan University, Hubei Key Laboratory of Medical Technology on Transplantation, Wuhan, Hubei 430071; \\ ${ }^{2}$ Department of General Surgery, The First Affiliated Hospital of Nanchang University, Nangchang, Jiangxi 330006; \\ ${ }^{3}$ Research Center of National Health Ministry on Transplantation Medicine Engineering and Technology, \\ The Third Xiangya Hospital of Central South University, Changsha, Hunan 410013, P.R. China
}

Received September 8, 2019; Accepted May 7, 2020

DOI: $10.3892 / \mathrm{mmr} .2020 .11312$

\begin{abstract}
Hepatic ischemia/reperfusion injury (HIRI) is a complex pathophysiological process that often leads to poor clinical prognosis. Clinically, the effective means to alleviate HIRI are limited. The aim of the present study was to investigate whether Alda-1, an activator of mitochondrial aldehyde dehydrogenase 2 (ALDH2), had a protective effect on HIRI and to investigate the mechanisms underlying this protective effect. Sprague-Dawley rats were treated with Alda-1 or Daidzin, an ALDH2 inhibitor, 30 min before partial (70\%) warm liver ischemia to induce HIRI. The 48 rats were randomly divided into four groups: Sham, ischemia injury (IR), IR-Alda-1, and IR-Daidzin. After 6 and $24 \mathrm{~h}$ of reperfusion, serum and liver tissue samples were collected and stored
\end{abstract}

Correspondence to: Dr Yanfeng Wang or Dr Qifa Ye, Zhongnan Hospital of Wuhan University, Institute of Hepatobiliary Diseases of Wuhan University, Transplant Center of Wuhan University, Hubei Key Laboratory of Medical Technology on Transplantation, 169 Donghu Road, Wuhan, Hubei 430071, P.R. China

E-mail: yanfengwang@whu.edu.cn

E-mail: yqf_china@163.com

${ }^{*}$ Contributed equally

Abbreviations: 4-HNE, 4-hydroxy-2-nonenal; ALDH2, aldehyde dehydrogenase 2; ALT, alanine aminotransferase; AST, aspartate aminotransferase; H\&E, hematoxylin \& eosin; HIRI, hepatic ischemia/reperfusion injury; HMGB1, high mobility group box 1 ; IFN- $\gamma$, interferon- $\gamma$; IL, interleukin; IP, intraperitoneal injection; MDA, malondialdehyde; mTOR, mammalian target of rapamycin; ROS, reactive oxygen species; $\mathrm{TNF}-\alpha$, tumor necrosis factor- $\alpha$; TLR4, toll-like receptor 4

Key words: ALDH2, Alda-1, hepatic ischemia/reperfusion injury, 4-HNE, autophagy for further experiments. Alanine aminotransferase, aspartate aminotransferase and hematoxylin \& eosin staining was used to evaluate the liver damage. Western blotting and reverse transcription-quantitative PCR were used to detect the expression of related proteins and mRNA. TUNEL staining was used to observe the apoptosis of liver cells. Transmission electron microscopy was used to detect the mitochondrial injuries. Alda-1 pretreatment ameliorated the HIRI-induced damage to the liver function and reduced histological lesions. Alda-1 also increased ALDH 2 activity after HIRI. Moreover, the pretreatment with Alda-1 reduced the accumulation of toxic aldehyde 4-hydroxy-2-nonenal, decreased the production of reactive oxygen species and malondialdehyde, reversed the damage to the liver mitochondria, attenuated hepatocyte apoptosis and inhibited the HIRI-induced inflammatory response, including high-mobility group box 1/toll-like receptor 4 signaling. Alda-1 also induced autophagy by upregulating autophagy-related 7 and Rab7 increasing the microtubule associated protein 1 light chain $3 \alpha \mathrm{II} / \mathrm{I}$ ratio and inhibiting p62 expression. ALDH2-induced autophagy was dependent on the activation of the AKT/mammalian target of rapamycin (mTOR) and AMP-activated protein kinase (AMPK) signaling pathways. In conclusion, the findings of the present study suggested that Alda-1 may protect the liver against HIRI-induced damage, including hepatic enzyme injury, acetaldehyde accumulation, oxidative stress, hepatocyte apoptosis and inflammation. Alda-1 may confer this protection by inducing autophagy through the AKT/mTOR and AMPK signaling pathways. Therefore, ALDH2 could represent a potential pharmacological target in the clinical treatment of HIRI.

\section{Introduction}

Hepatic ischemia/reperfusion injury (HIRI) often occurs as a consequence of liver surgery and numerous types of injury, including transplantation, trauma, shock and resection (1). HIRI has been discovered to be a major cause of liver dysfunction and organ rejection (2). Therefore, the identification of 
pharmacological treatments that effectively improve HIRI outcomes remains a priority over invasive methods.

HIRI usually involves initial ischemia-induced cell damage, followed by a reperfusion-induced inflammatory response, which directly injures the liver tissue (3). As the primary producers of ATP and reactive oxygen species (ROS), hepatocyte mitochondria are critical for normal liver function (4). Therefore, clinical interventions targeting the functional homeostasis of liver mitochondria represent a promising approach for HIRI treatment.

Previous studies using experimental models have demonstrated that aldehyde dehydrogenase 2 (ALDH2) served a pivotal role in the pathophysiology of cardiovascular injury $(5,6)$. ALDH 2 is the primary enzyme that catalyzes ethanol metabolism by detoxifying acetaldehyde into non-toxic acetate; acetate is then generated in the mitochondrial matrix (7). In particular, ALDH2 is known have an important role in the elimination of endogenous oxidized aldehyde adducts (8). These adducts, which are formed by lipid peroxidation under oxidative stress, include 4-hydroxy-2-nonenal (4-HNE) and malondialdehyde (MDA) $(8,9)$. The excessive accumulation of the 4-HNE adduct was discovered to lead to mitochondrial dysfunction during acute myocardial IRI and chronic heart failure, which impaired the cardiac contractility, mitochondrial bioenergetics and redox balance $(5,10)$. Interestingly, these detrimental outcomes were found to be reversed through the upregulation of ALDH2 (11). Conversely, 4-HNE and ROS have been identified to inhibit ALDH2 activity (11).

Guo et al (12) previously demonstrated that ALDH2 was activated by Alda-1, improving chronic alcohol-induced steatosis and apoptosis. Alda-1, a member of the ALDH2 isozyme-specific activator family, was discovered to increase the catalytic activity of ALDH2 both in vivo and in vitro (13). However, whether ALDH2 activation can exert a protective effect during HIRI remains unclear.

Autophagy is an intracellular degradative process that targets damaged organelles, dysfunctional proteins and harmful products through the formation of autophagosomes and autolysosomes (14). Autophagy has also been associated with the maintenance of functional liver homeostasis (14); however, the effects of autophagy on HIRI remain controversial. Previous studies have indicated that ALDH2 served a protective role in myocardium IRI and alcohol-induced chronic hepatic steatosis by inducing autophagy $(6,12)$. However, to the best of our knowledge, the potential underlying crosstalk between ALDH2 and autophagy in HIRI have not yet been characterized.

Therefore, the aim of the present study was to evaluate the effects of Alda-1 on HIRI and to examine the underlying mechanisms. It was hypothesized that Alda-1 may attenuate HIRI by increasing ALDH2 activity, reducing oxidative stress and apoptosis, inhibiting the inflammatory response and regulating autophagy.

\section{Materials and methods}

Experimental design and animal model establishment. A total of 48 male inbred Sprague Dawley rats (weight, 250-300 g) aged 7-8 weeks were obtained from Beijing Vital River Laboratory Animal Technology Co., Ltd. Rats were raised in the Animal
Experiment Center of The Zhongnan Hospital of Wuhan University (Wuhan, China). All rats were maintained under standard animal care conditions at $24 \pm 3^{\circ} \mathrm{C}$ and $60 \%$ humidity, with a 12-h dark/light cycle and free access to food and water. All animal experiments and protocols were approved by the Committee on the Experimental Animal Regulations of the Zhongnan Hospital of Wuhan University (approval no. A237; Wuhan, China) and conformed to the Guide for the Care and Use of Laboratory Animals (15).

To investigate the effects of Alda-1 on HIRI, rats were treated intraperitoneally $30 \mathrm{~min}$ before warm liver ischemia with either $10 \mathrm{mg} / \mathrm{kg}$ Alda-1 (cat. no. HY-18936; MedChemExpress) (16) or $100 \mathrm{mg} / \mathrm{kg}$ Daidzin (an ALDH2 inhibitor; cat. no. HY-N0018; MedChemExpress). Both Alda-1 and Daidzin were dissolved in 0.1\% DMSO (Sigma-Aldrich; Merck KGaA). A total of 48 rats were divided into four groups ( $n=12 /$ group): i) A sham group, in which rats were given laparotomies without HIRI; ii) an IR group, where rats were treated with vehicle $(0.1 \%$ DMSO) 30 min before laparotomy with HIRI; iii) an IR-Alda-1 Group, where rats were treated with $10 \mathrm{mg} / \mathrm{kg}$ Alda-1 $30 \mathrm{~min}$ before laparotomy with HIRI; and iv) an IR-Daidzin group, where rats were treated with $100 \mathrm{mg} / \mathrm{kg}$ Daidzin $30 \mathrm{~min}$ before laparotomy with HIRI (Fig. 1A).

Briefly, rats were fasted for $12 \mathrm{~h}$ before the experiment but allowed free access to water. Rats were then anesthetized with intraperitoneal (IP) injection of $50 \mathrm{mg} / \mathrm{kg}$ sodium pentobarbital. Normal body temperature was maintained using a heating pad at $37 \pm 0.5^{\circ} \mathrm{C}$ and was monitored with a rectal probe. Under anesthesia, an abdominal midline laparotomy was performed on all rats. Partial (70\%) liver ischemia was induced in the HIRI groups by clamping the median and left lobes for 60 min with a microvascular clip. Hepatic ischemia was confirmed by color changes in the tissue (Fig. 1B). After the 60 min period of warm ischemia, the clamp was removed, the abdomen was then closed, and the rats were allowed to recover in a warm environment. No rats died within $24 \mathrm{~h}$ of the laparotomy and no antibiotics were administrated over the course of the experiment.

After 6 or 24 h of reperfusion, rats were sacrificed using $100 \mathrm{mg} / \mathrm{kg}$ pentobarbital sodium (IP). After cessation of breathing and heartbeat, $5 \mathrm{ml}$ blood samples were collected through inferior vena cava punctures using K2-EDTA test tubes, then centrifuged at $3,000 \mathrm{x}$ g for $15 \mathrm{~min}$ at $4^{\circ} \mathrm{C}$ to extract serum. Liver tissue samples were harvested from the median and left lobes of the ischemic liver. Serum and liver tissue samples were frozen in liquid nitrogen, then stored at $-80^{\circ} \mathrm{C}$. A section of each liver tissue sample was immersed in $4 \%$ paraformaldehyde at $25^{\circ} \mathrm{C}$ for $48 \mathrm{~h}$ for histopathology.

Analysis of liver enzymes and serum analytes. The levels of alanine aminotransferase (ALT) and aspartate aminotransferase (AST) released into the serum were detected using an ALT ELISA kit (cat. no. E-BC-K235-M; Elabscience Biotechnology, Co., Ltd.) and an AST ELISA kit (cat. no. E-BC-K236-M; Elabscience Biotechnology, Co., Ltd.) following the protocols of the clinical laboratory of the Zhongnan Hospital of Wuhan University. The serum levels of tumor necrosis factor- $\alpha$ (TNF- $\alpha)$, interleukin (IL)-1 $\beta$, IL-6 and 4-HNE in the serum were measured using 
A

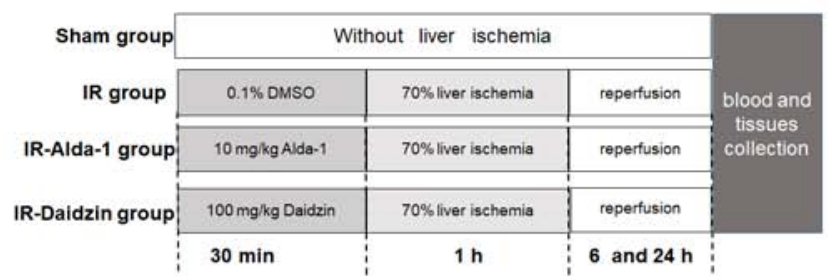

B

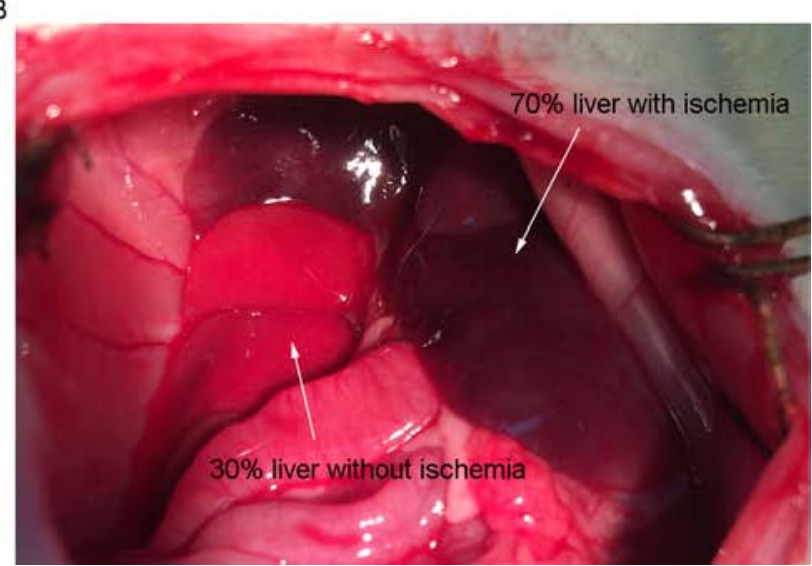

Figure 1. Experimental design and animal model establishment. (A) Experimental groupings used in the present study. (B) 70\% liver IR injury model establishment in rats. After $1 \mathrm{~h}$ liver ischemia, the color of the ischemic liver (70\%) is dark red, while the color of the liver without ischemia (30\%) is bright red. IR, ischemia/reperfusion.

TNF- $\alpha$ ELISA kit (cat. no. E-EL-R0019c), IL-1 $\beta$ ELISA kit (cat.no.E-EL-R0012c), IL-6 ELISA kit (cat.no. E-EL-R0015c) and 4-HNE ELISA kit (cat. no. E-EL-0128c; all from Elabscience Biotechnology, Co., Ltd.) according to the manufacturers' protocols.

Histopathology. The samples after paraffin immersion were embedded in paraffin wax and cut into 5- $\mu \mathrm{m}$ sections for histological analysis via hematoxylin-eosin staining (HE; hematoxylin staining for 5-15 min and eosin staining for 1-3 min; all performed at room temperature). The degree of liver injury was assessed according to Suzuki's grading scale (17): i) Grade 0, no congestion, no vacuolization and no necrosis; ii) grade 1, minimal congestion, minimal vacuolization and single-cell necrosis; iii) grade 2, mild congestion, mild vacuolization and $<30 \%$ necrosis; iv) grade 3 , moderate congestion, moderate vacuolization and $\leq 60 \%$ necrosis; and v) grade 4 , severe congestion, severe vacuolization and $>60 \%$ necrosis. Two blinded pathologists independently evaluated the sections under a light microscope (magnification, x100).

Determination of ALDH2 activity. ALDH2 activity was measured using an ALDH2 activity assay kit (cat.no. A075-1-1; Nanjing Jiancheng Bioengineering Institute), according to the manufacturer's protocol. Briefly, the activity of the ALDH2 enzyme was determined by monitoring the conversion of $\mathrm{NAD}^{+}$to $\mathrm{NADH}$ at a spectrophotometric absorbance of $340 \mathrm{~nm}$. ALDH2 activity is expressed as nmol NADH/min per mg protein.

Oxidative stress analysis. The levels of 4-HNE (cat. no. H268), MDA (cat. no. A003-1-2) and ROS (cat. no. E004-1-1) in liver tissue samples were measured using specific rat colorimetric assay kits (Nanjing Jiancheng Bioengineering Institute), according to the manufacturers' protocol.

Western blotting. Western blotting was performed as previously described (18). Briefly, total protein from liver tissues was extracted using RIPA lysis buffer (Beyotime Institute of Biotechnology), supplemented with protease inhibitor and phosphatase inhibitor (Roche Applied Science). Protein concentration of the supernatant was determined by the bicinchoninic acid kit (cat. no. G2026; Wuhan Servicebio Technology Co., Ltd.). Subsequently, $50 \mu \mathrm{g}$ protein/lane was loaded and separated by SDS-PAGE on 8-12\% gels, then transferred onto PVDF membranes. The PVDF membranes were blocked with $5 \%$ nonfat milk for $1 \mathrm{~h}$ at room temperature and incubated overnight at $4{ }^{\circ} \mathrm{C}$ with one of the following primary antibodies: Rabbit anti-ALDH2 (1:1,000; cat. no. 15310-1-AP; ProteinTech Group, Inc.), rabbit anti-4-HNE (1:500; cat. no. ab46545; Abcam), rabbit anti-high mobility group box 1 (HMGB1; 1:1,000; cat. no. 10829-1-AP; ProteinTech Group, Inc.), rabbit anti-toll-like receptor 4 (TLR4; 1:1,000; cat. no. 19811-1-AP; ProteinTech Group, Inc.), rabbit anti-beclin1 (1:500; cat. no. 11306-1-AP; ProteinTech Group, Inc.), rabbit anti-autophagy-related 7 (ATG7; 1:1,000; cat. no. 67341-1-IG; ProteinTech Group, Inc.), rabbit anti-p62 (1:1,000; cat. no. 18420-1-AP; ProteinTech Group, Inc.), rabbit anti-Rab7 (1:1,000; cat. no. 55469-1-AP; ProteinTech Group, Inc.), rabbit anti-microtubule associated protein 1 light chain 3 $\alpha$ (LC3; 1:500; cat. no. 14600-1-AP; ProteinTech Group, Inc.), mouse anti-AKT (1:1,000; cat. no. 10176-2-AP; ProteinTech Group, Inc.), rabbit anti-phosphorylated (p-)AKT at Thr308 (1:1,000; cat. no. 13038T; Cell Signaling Technology, Inc.), rabbit anti-mTOR (1:300; cat. no. 20657-1-AP; ProteinTech Group, Inc.), rabbit anti-p-mTOR at Ser2448 (1:1,000; cat. no. 2971S; Cell Signaling Technology, Inc.), rabbit anti-S6 (1:1,000; cat. no. 14823-1-AP; ProteinTech Group, Inc.), rabbit anti-p-S6 at Ser240/244 (1:1,000; cat. no. 5364T; Cell Signaling Technology, Inc.), mouse anti-AMP-activated protein kinase (AMPK; 1:1,000; cat. no. 2793S; Cell Signaling Technology, Inc.), rabbit anti-p-AMPK at Thr172 (1:1,000; cat. no. 2535T; Cell Signaling Technology, Inc.) or rabbit anti- $\beta$-actin (1:1,000; cat. no. 20536-1-AP; ProteinTech Group, Inc.). The following day, the PVDF membranes were washed three times and incubated with goat anti-mouse (1:2,000; cat. no. SA00001-1, ProteinTech Group, Inc.) or goat anti-rabbit (1:2,000; cat. no. SA00001-2, ProteinTech Group, Inc.) horseradish peroxidase (HRP)-conjugated secondary antibodies for $2 \mathrm{~h}$ at room temperature. Finally, bands were detected using 
a chemiluminescent ECL reagent (cat. no. G2020; Wuhan Servicebio Technology Co., Ltd.). Protein expression bands were quantified using densitometry analysis with ImageJ v6.0 software (National Institutes of Health) and normalized to $\beta$-actin.

Immunohistochemistry staining and immunofluorescence. Hepatic HMGB1 and TLR4 expression levels were also evaluated by immunohistochemical staining. Briefly, paraffin-embedded 5- $\mu \mathrm{m}$ liver tissue sections were treated with $3 \%$ hydrogen peroxide for $15 \mathrm{~min}$ at $37^{\circ} \mathrm{C}$ to block endogenous peroxidase activity. Nonspecific binding was blocked by incubating the sections with 5\% BSA (Wuhan Goodbio Technology Co. Ltd.) for $20 \mathrm{~min}$ at room temperature. Liver tissue sections were then incubated with a polyclonal rabbit anti-HMGB1 (1:100; cat. no. 10829-1-AP; ProteinTech Group, Inc.) antibody or anti-TLR4 (1:200; cat. no. 19811-1-AP; ProteinTech Group, Inc.) antibody at $4{ }^{\circ} \mathrm{C}$ overnight, followed by incubation with polymer-HRP-conjugated anti-rabbit IgG (1:1,000; cat. no. SA00001-2, ProteinTech Group, Inc.) for $30 \mathrm{~min}$ at room temperature. Lastly, slides were stained for $3 \mathrm{~min}$ at room temperature using a diaminobenzidine (DAB) kit with HRP as the substrate. Finally, the sections were dehydrated in a graded ethanol solution and cleared with xylene. The HMGB1 and TLR4 protein expression levels were viewed under a light microscope (magnification, x200).

For immunofluorescence, Liver tissue were fixed, embedded and sectioned using the same method mentioned above. Sections were blocked in 5\% BSA (Beijing Solarbio Science \& Technology Co., Ltd.) at room temperature for $2 \mathrm{~h}$ and were incubated with rabbit anti-IL-6 (cat. no. 21865-1-AP; 1:100; ProteinTech Group, Inc.) overnight at $4^{\circ} \mathrm{C}$. The sections were then incubated with Alexa Fluor 555-conjugated goat anti-rabbit (cat. no. P0179; 1:100; Shanghai Biyuntian Biological Co., Ltd.) and DAPI (cat. no. C1002; Shanghai Biyuntian Biological Co., Ltd.) at room temperature for $1 \mathrm{~h}$. Results were visualized using a fluorescence microscope (magnification, x400) and images were analyzed by ImageJ software (version 1.51; National Institutes of Health).

Reverse transcription-quantitative PCR (RT-qPCR). Total RNA was extracted from $100 \mathrm{mg}$ frozen liver tissue samples using TRIzol ${ }^{\circledR}$ reagent (Invitrogen; Thermo Fisher Scientific, Inc.), $20 \%$ chloroform and 50\% isopropyl alcohol. Total RNA was subsequently reverse transcribed into cDNA using a reverse transcription kit (Thermo Fisher Scientific, Inc.), according to the manufacturer's protocol. RT was performed at $42^{\circ} \mathrm{C}$ for $1 \mathrm{~h}$ followed by an incubation at $75^{\circ} \mathrm{C}$ for $5 \mathrm{~min}$. The thermocycling conditions were as follows: $50^{\circ} \mathrm{C}$ for $2 \mathrm{~min}, 95^{\circ} \mathrm{C}$ for $10 \mathrm{~min}$, followed by 40 cycles of $95^{\circ} \mathrm{C}$ for $10 \mathrm{sec}$ and $60^{\circ} \mathrm{C}$ for $30 \mathrm{sec}$. RT-PCR was subsequently performed using SYBR Green (Shanghai Yeasen Biotechnology Co. Ltd.) to determine the expression levels of target genes. The relative expression of genes was calculated using the $2^{-\Delta \Delta C q}$ method. Gene expression was normalized against that of the $\beta$-actin gene (19). All primers used for the RT-qPCR are listed in Table I.

TUNEL staining. The liver tissue was fixed in $4 \%$ paraformaldehyde solution at room temperature for $24 \mathrm{~h}$, paraffin-embedded, dehydrated in a graded ethanol series and coronally cut into
Table I. Nucleotide sequences of primers used for reverse transcription-quantitative PCR.

\begin{tabular}{ll} 
Gene & \multicolumn{1}{c}{ Primer sequence $\left(5^{\prime} \rightarrow 3^{\prime}\right)$} \\
\hline $\begin{array}{l}\text { High mobility } \\
\text { group box } 1\end{array}$ & F: GGCGGCTGTTTTGTGACAT \\
Toll-like & R: ACCCAAAATGGGCAAAAGCA \\
receptor 4 & F: TGTATCGGTGGTCAGTGTGC \\
Interferon- $\gamma$ & R: CAGCTCGTTTCTCACCCAGT \\
& F: GAGGAACTGGCAAAAGGACG \\
IL-1 $\beta$ & R: AGGTGCGATTCGATGACACT \\
& F: GAGGCTGACAGACCCCAAAAGA \\
IL-6 & R: TCCACAGCCACAATGAGTGACA \\
& F: AGCGATGATGCACTGTCAGA \\
Tumor & R: GGAACTCCAGAAGACCAGAGC \\
necrosis- $\alpha$ & F: GTGATCGGTCCCAACAAGGA \\
$\beta$ - actin & R: TTTGCTACGACGTGGGCTAC \\
& F: TGCTATGTTGCCCTAGACTTCG \\
& R: GTTGGCATAGGTCTTTACGG
\end{tabular}

IL, interleukin; F, forward; R, reverse.

$5 \mu \mathrm{m}$ sections. Sections were deparaffinized using xylene and rehydrated in a descending ethanol series. Tunel assay (Roche Diagnostics) was used to detect quantitatively the apoptotic hepatocytes according to the manufacturer's protocol. Liver tissue sections were incubated with fluorescein-dUTP at $37^{\circ} \mathrm{C}$ for $1 \mathrm{~h} .6$ visual fields were randomly selected under a fluorescence microscope. The normal hepatocytes were stained blue and the apoptosis-positive cells green. Total hepatocytes and TUNEL-positive cells were counted under a fluorescence microscope (magnification, $\mathrm{x} 200$ ). The apoptotic rate was calculated as: (The number of TUNEL-positive cells/the total number of hepatocytes) $\mathrm{x} 100 \%$. Analysis was carried out using Image Pro Plus 6.0 software (Media Cybernetics, Inc.).

Transmission electron microscopy (TEM). The 1-2 $\mathrm{mm}^{3}$ fresh liver tissue fixed in $2.5 \%$ glutaraldehyde solution was rinsed in PBS and fixed with $1 \%$ osmium tetroxide for $2 \mathrm{~h}$ at room temperature. Following dehydration with a graded acetone series and embedding in Pon812 epoxy resin for $12 \mathrm{~h}$ at room temperature, the liver tissue was cut into $1 \mu \mathrm{m}$ sections. The sections were stained with $3 \%$ uranyl acetate at room temperature for $30 \mathrm{~min}$ and washed with double distilled water. Then, the sections were stained with $3 \%$ lead citrate at room temperature for $10 \mathrm{~min}$ and washed with double distilled water. The mitochondrial injuries was observed by Tecnai $\mathrm{G}^{2}$ 20 Twin TE microscope (FEI; Thermo Fisher Scientific, Inc.) at room temperature: 5 fields were selected for each specimen for statistical analysis.

Statistical analysis. Statistical analysis was performed using SPSS 16.0 statistical software (SPSS, Inc.). Data are presented as the mean $\pm \mathrm{SD}$. For experimental data following a normal distribution, statistical differences were determined using an ANOVA, followed by a Tukey's post-hoc test. Otherwise, nonparametric data were analyzed using the Kruskal-Wallis 


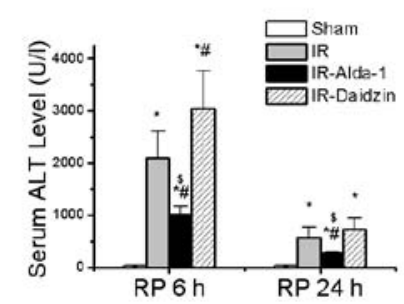

B

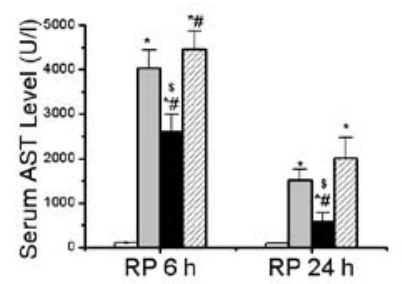

C

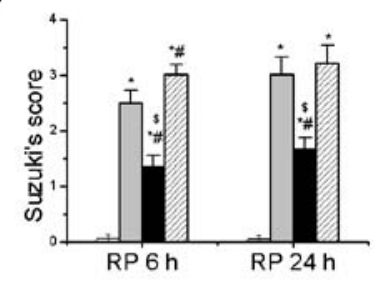

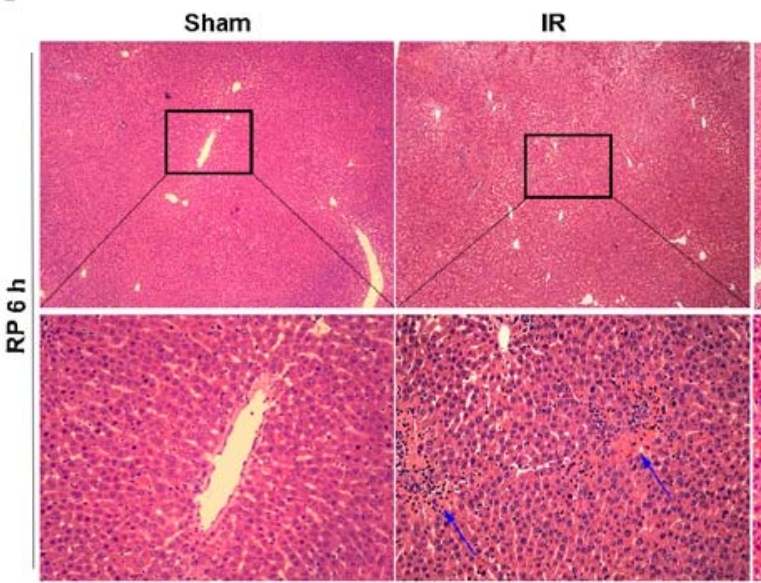
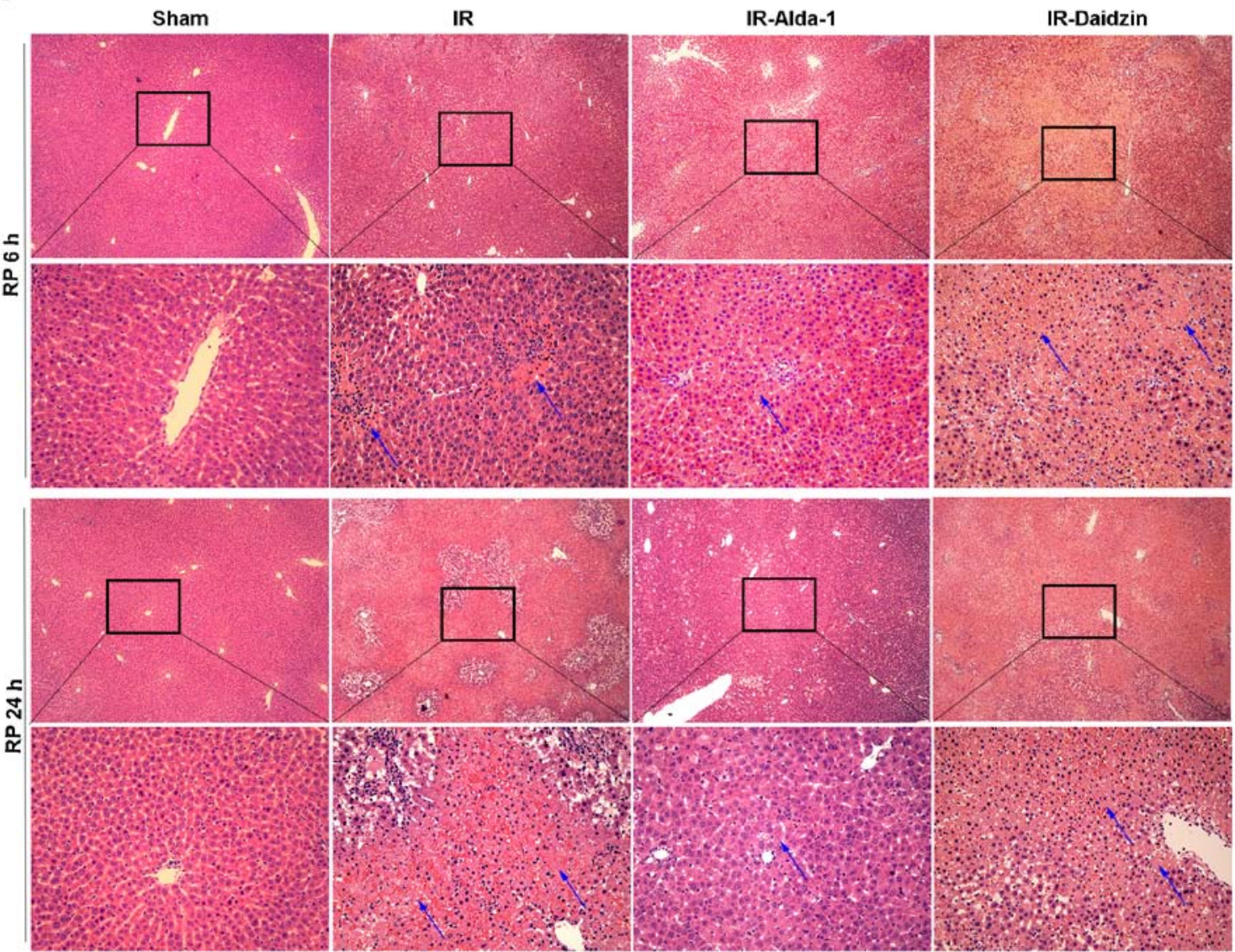

Figure 2. Alda-1 pretreatment ameliorates HIRI in rats. The serum levels of (A) ALT and (B) AST in each group after 6 and $24 \mathrm{~h}$ of reperfusion. (C) Suzuki's scores (17) for the H\&E-stained HIRI rat livers of each group. (D) Representative images of HIRI rat livers after 6 and $24 \mathrm{~h}$ of reperfusion stained with H\&E. Blue arrows indicate hepatocellular necrosis. Scale bars, 100 and $400 \mu \mathrm{m}$. Data are presented as the mean $\pm \mathrm{SD}$. $\mathrm{n}=6$. ${ }^{*} \mathrm{P}<0.05$ vs. the sham group; ${ }^{*} \mathrm{P}<0.05 \mathrm{vs}$. the IR group; ${ }^{\mathrm{P}} \mathrm{P}<0.05$ vs. the IR-Daidzin group. HIRI, hepatic ischemia/reperfusion injury; IR, ischemia/reperfusion, ALT, alanine aminotransferase; AST, aspartate aminotransferase; H\&E, hematoxylin \& eosin; RP, reperfusion.

test, followed by a Dunn's post-hoc test. $\mathrm{P}<0.05$ was considered to indicate a statistically significant difference.

\section{Results}

Alda-1 pretreatment ameliorates HIRI in rats. After $70 \%$ warm hepatic ischemia for $60 \mathrm{~min}$, followed by 6 or $24 \mathrm{~h}$ of reperfusion, the serum levels of ALT and AST were significantly increased in the IR group compared with the sham group (Fig. 2A and B). However, the pretreatment with Alda-1 prior to HIRI decreased the serum levels of ALT and AST after 6 and $24 \mathrm{~h}$ of reperfusion compared with the IR and IR-Daidzin groups. These results were supported by the histological lesions observed in the liver tissues with H\&E staining (Fig. 2C and D). Extensive necrosis (indicated by the white arrows), congestion and neutrophil infiltration were observed in the IR and IR-Daidzin groups after 6 and $24 \mathrm{~h}$ of reperfusion. These lesions were significantly reduced in the IR-Alda-1 group compared with the IR group, based on
Suzuki's HIRI scoring system (17). These results suggested that ALDH2 may serve a role in HIRI and that ALDH2 activation may confer protection from HIRI.

ALDH2 activity and expression in HIRI model rats. Changes in the ALDH2 expression levels were then evaluated in HIRI rats. ALDH2 protein expression levels were not significantly different among the four groups at either 6 or $24 \mathrm{~h}$ (Fig. 3A and B). However, HIRI significantly decreased ALDH2 activity in the IR group compared with rats in the sham group at both 6 and $24 \mathrm{~h}$. This effect was partially rescued by ALDH2 activation in the IR-Alda-1 group (Fig. 3C).

Effects of Alda-1 on 4-HNE accumulation and oxidative stress in HIRI model rats. The accumulation of ROS, MDA and 4-HNE aldehydes has been discovered to promote toxic effects associated with HIRI $(4,8,9)$. A significant increase in 4-HNE protein expression levels, serum levels and tissue 


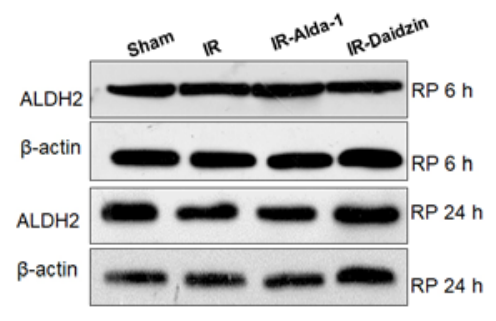

B

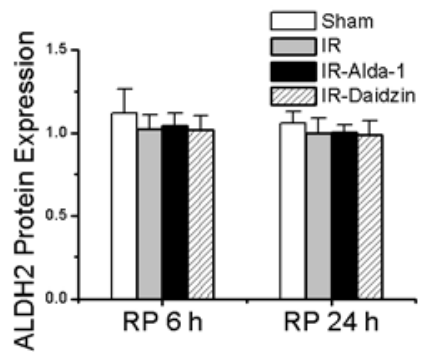

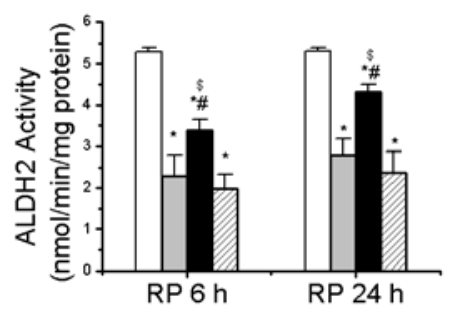

Figure 3. Liver ALDH2 expression in rats with hepatic IR injury. (A) Western blotting was used to analyze ALDH2 expression levels in liver tissue after 6 and $24 \mathrm{~h}$ of reperfusion. Relative expression was normalized to the $\beta$-actin loading control. (B) Densitometric analysis of ALDH2 protein expression levels from part (A). (C) ALDH2 activity after $24 \mathrm{~h}$ of reperfusion in each group. Data are presented as the mean $\pm \mathrm{SD} . \mathrm{n}=6 .{ }^{*} \mathrm{P}<0.05$ vs. the sham group; ${ }^{*} \mathrm{P}<0.05 \mathrm{vs}$. the IR group; ${ }^{\mathrm{P}} \mathrm{P}<0.05$ vs. the IR-Daidzin group. ALDH2, aldehyde dehydrogenase 2; IR, ischemia/reperfusion; RP, reperfusion.

A

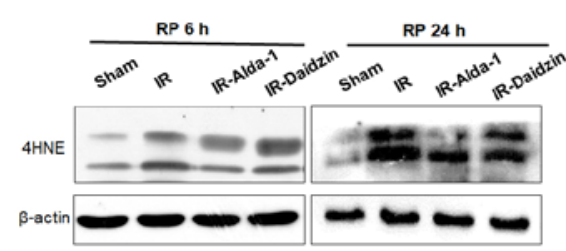

$\mathrm{D}$

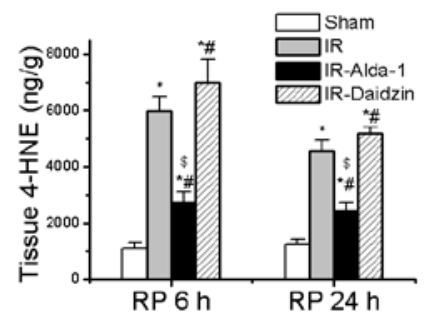

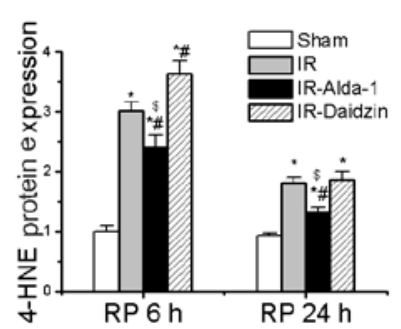

$\mathrm{E}$

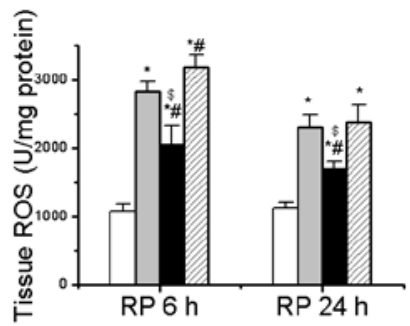

C
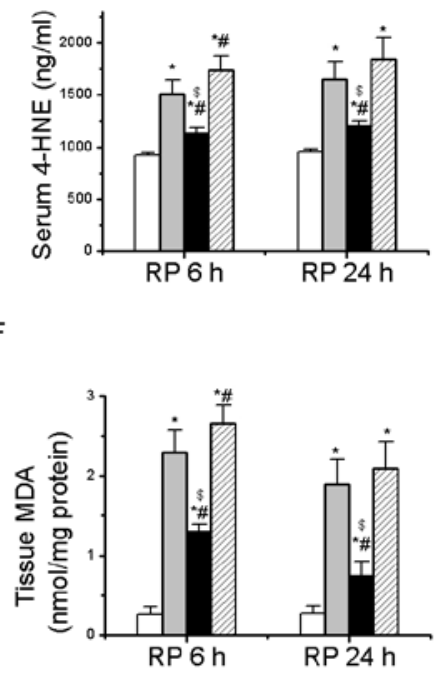

Figure 4. Effect of Alda-1 on 4-HNE accumulation and oxidative stress in rats with hepatic IR injury. (A) Western blotting was used to analyze the formation of 4-HNE protein adducts in liver tissue after 6 and $24 \mathrm{~h}$ of reperfusion. Relative expression was normalized to the $\beta$-actin loading control. (B) Densitometric semi-quantification of 4-HNE protein adduct formation from part (A). (C) Serum levels of 4-HNE after 6 and $24 \mathrm{~h}$ of reperfusion. (D) 4-HNE, (E) ROS levels and (F) MDA levels in liver tissue after 6 and $24 \mathrm{~h}$ of reperfusion. Data are presented as the mean \pm SD. $n=6 .{ }^{*} \mathrm{P}<0.05$ vs. the sham group; ${ }^{*} \mathrm{P}<0.05$ vs. the IR group; ${ }^{~} \mathrm{P}<0.05$ vs. the IR-Daidzin group. RP, reperfusion; 4-HNE, 4-HNE, 4-hydroxy-2-nonenal; ROS, reactive oxygen species; MDA, malondialdehyde; IR, ischemia/reperfusion.

accumulation was observed following IR and 6 or $24 \mathrm{~h}$ reperfusion compared with the sham group (Fig. 4A-D), while these increases were partially reversed in the IR-Alda-1 group. To investigate the relationship between the ALDH2-mediated liver protection and oxidative stress, the levels of ROS and MDA were measured in the liver tissue; Alda- 1 pretreatment reduced the production of ROS and MDA in hepatic tissues following 6 and $24 \mathrm{~h}$ of reperfusion compared with the IR and IR-Daidzin groups (Fig. 4E and F).

Alda-1 pretreatment improves liver mitochondrial damage and attenuates hepatocyte apoptosis in HIRI model rats. Mitochondrial changes in the hepatocytes were observed after $24 \mathrm{~h}$ of reperfusion using TEM. Substantial mitochondrial damage occurred in the IR group hepatocytes compared with the sham group (Fig. 5A). However, decreased mitochondrial edema was observed in the IR-Alda-1 group (indicated in the figure by arrows). TUNEL staining also indicated that Alda-1 pretreatment attenuated hepatocyte apoptosis $24 \mathrm{~h}$ after reperfusion compared with the IR and IR-Daidzin groups (Fig. 5B and C).
Alda-1 pretreatment inhibits inflammatory responses in HIRI model rats. HMGB1 and TLR4 have been reported to be involved in inflammatory responses associated with HIRI (4). After 6 and $24 \mathrm{~h}$ of reperfusion, the protein expression levels of HMGB1 and TLR4 were significantly upregulated in the IR group compared with the sham group (Fig. 6A-C). Meanwhile, Alda-1 pretreatment significantly inhibited the protein expression levels of HMGB1 and TLR4 compared with the IR group.

RT-qPCR was then used to analyze the mRNA expression levels of HMGB1, TLR4 and other downstream inflammatory factors. Alda-1 pretreatment significantly reduced the mRNA expression levels of HMGB1, TLR4, interferon- $\gamma(\mathrm{IFN}-\gamma)$, IL-1 $\beta$, IL- 6 and TNF- $\alpha$ at 6 and $24 \mathrm{~h}$ after reperfusion, compared with the mRNA expression levels of these genes in the IR and IR-Daidzin groups (Fig. 6D-I). Similar trends were also observed in the serum levels of IL-1 $\beta$, IL- 6 and TNF- $\alpha$ after 6 and $24 \mathrm{~h}$ of reperfusion (Fig. 6J-K). The results of the immunohistochemical staining of HMGB1 and TLR4 expression levels, and of IL-6 
A

A Sham
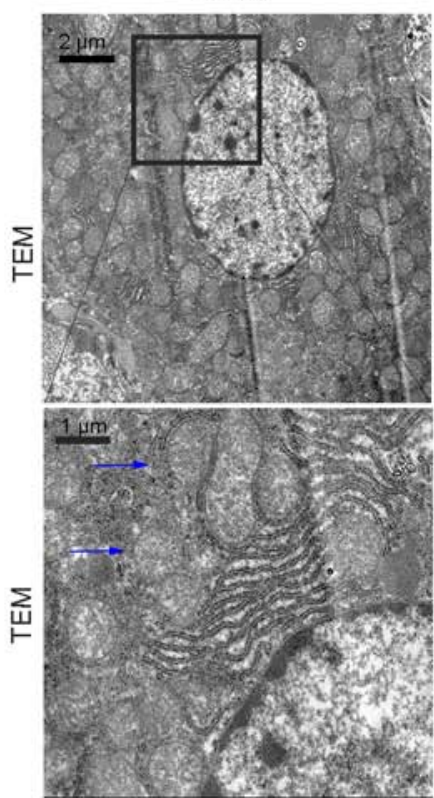

B

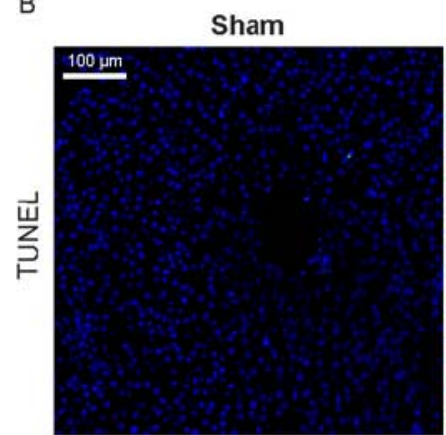

IR

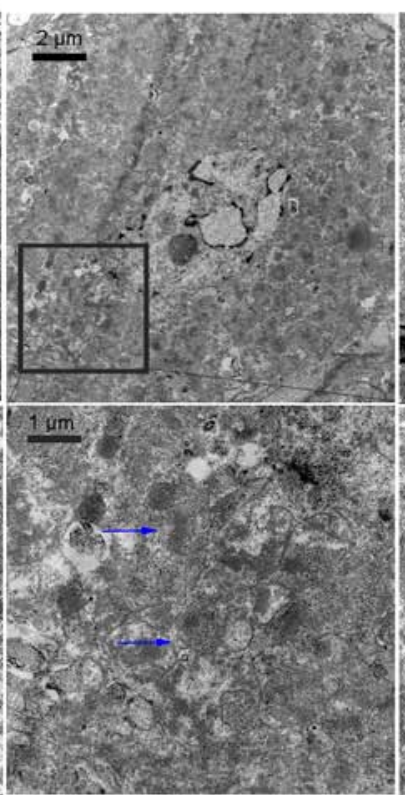

IR-Alda-1

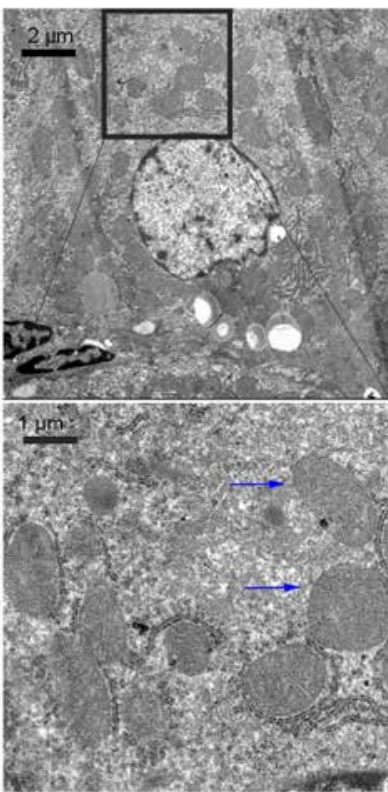

IR-Daidzin

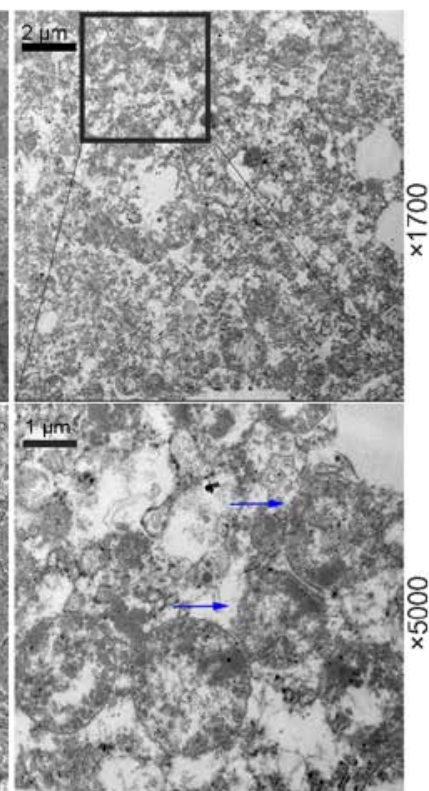

IR

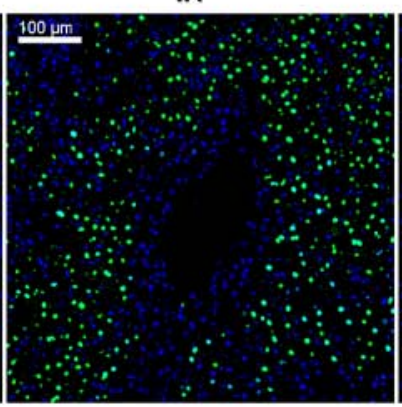

IR-Alda-1

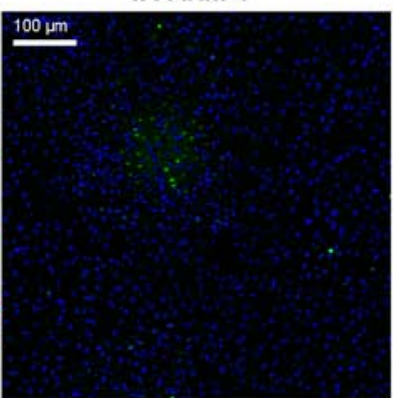

IR-Daidzin

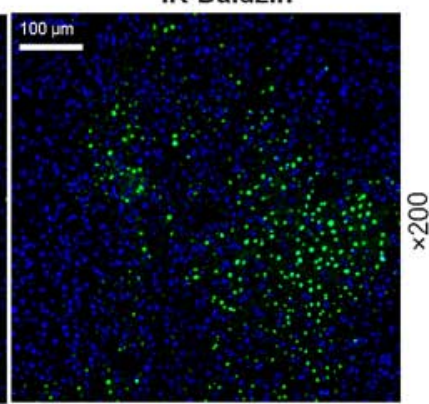

C

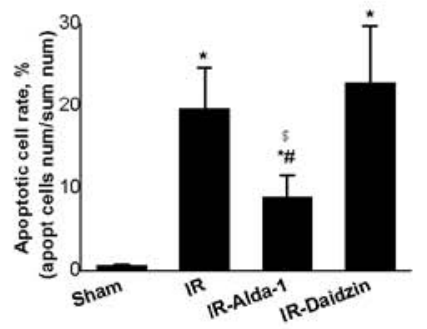

Figure 5. Alda-1 pretreatment reduces damage to liver mitochondria and hepatocyte apoptosis in HIRI model rats. (A) Representative transmission electron micrographs showing mitochondria in the liver tissue after $24 \mathrm{~h}$ of reperfusion. Blue arrows indicate mitochondria. Magnification, $\mathrm{x} 1,700$ and x5,000. Scale bars, 1 or $2 \mu \mathrm{m}$. (B) Representative TUNEL-stained images showing HIRI rat livers after $24 \mathrm{~h}$ of reperfusion. Magnification, $\mathrm{x} 200$; scale bar, $100 \mu \mathrm{m}$. (C) TUNEL-stained apoptotic cell rate of each group. Data are presented as the mean $\pm \mathrm{SD}$. $\mathrm{n}=6 .{ }^{*} \mathrm{P}<0.05$ vs. the sham group; ${ }^{*} \mathrm{P}<0.05$ vs. the IR group; ${ }^{\circledR} \mathrm{P}<0.05$ vs. the IR-Daidzin group. RP, reperfusion; HIRI, hepatic ischemia/reperfusion injury; IR, ischemia/reperfusion; TEM, transmission electron microscope.

immunofluorescence, were consistent with the western blotting, ELISA and RT-qPCR data (Fig. 7). Thus, these findings indicated that Alda-1 pretreatment may protect the liver against HIRI by inhibiting the HMGB1/TLR4 inflammatory pathway.

Alda-1 pretreatment restores HIRI-induced suppression of autophagy. To evaluate the effects of Alda-1 on HIRI-induced autophagy, the protein expression levels of Beclin1 (important for autophagy nucleation) (20), ATG7 (a mediator of autophagosome formation) (12), p62 (a specific protein adaptor degraded by autophagy) (20), Rab7 (a small GTPase protein that stimulates lysosomal biogenesis and autophagic vacuole maturation) (12) and LC3 (important for autophagolysosome formation) (12) were evaluated. The expression level of Beclin1 protein showed no significant differences between the groups (Fig. 8B). Compared with the sham group, the IR group had significantly downregulated expression levels of ATG7 and Rab7, a significantly reduced LC3II/I ratio and significantly upregulated protein expression levels of p62 after 6 and $24 \mathrm{~h}$ of reperfusion (Fig. 8A, C-F). These effects were significantly reversed in the IR-Alda-1 group (Fig. 8A, C-F). Thus, these 
A

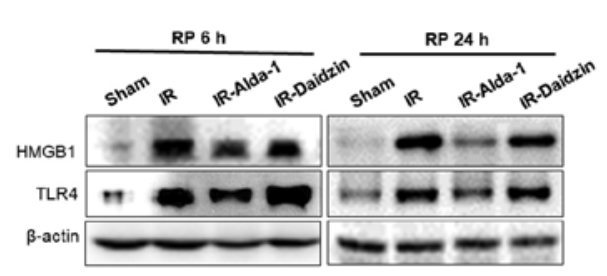

D

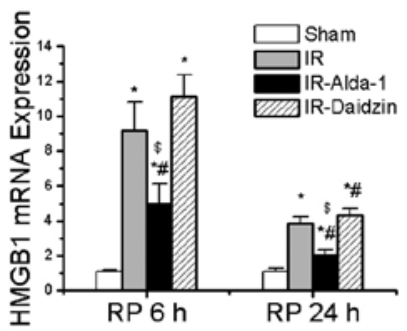

G
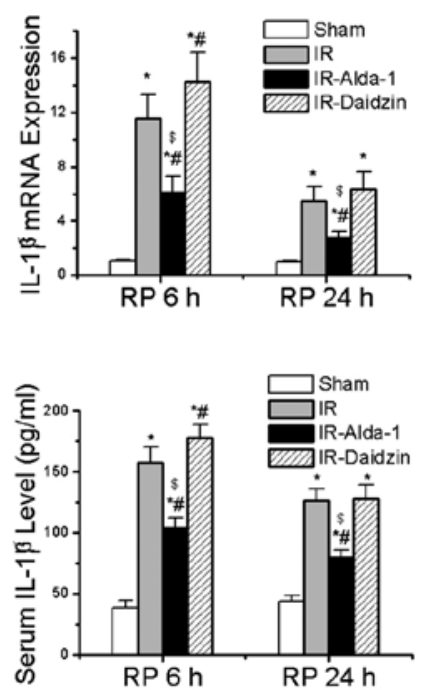

B

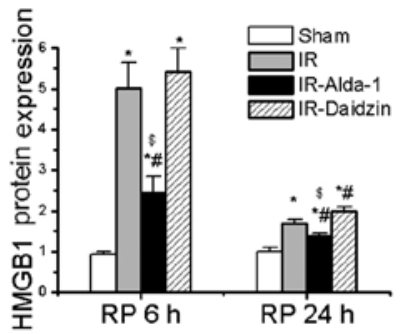

$\mathrm{E}$

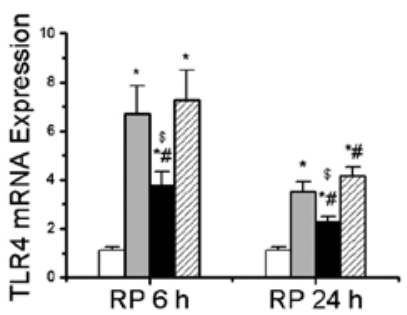

$\mathrm{H}$

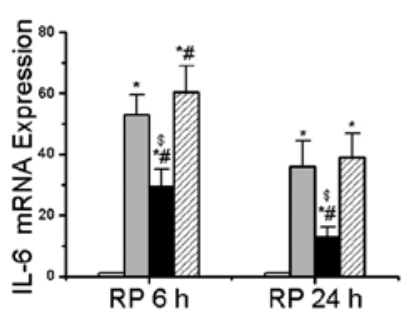

$\mathrm{K}$

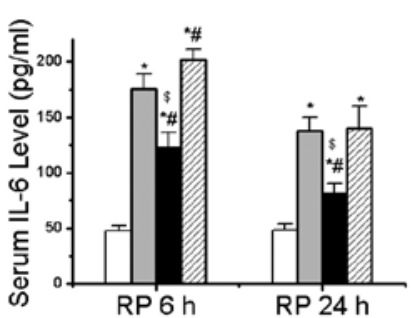

C

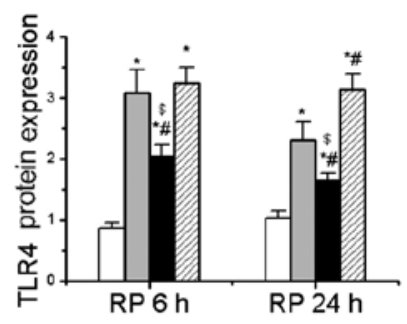

$\mathrm{F}$

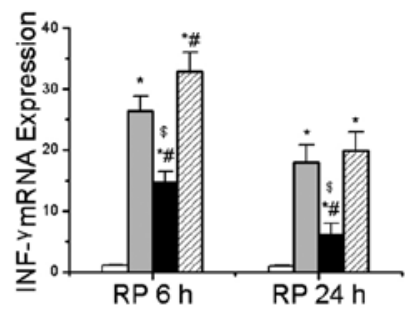

।

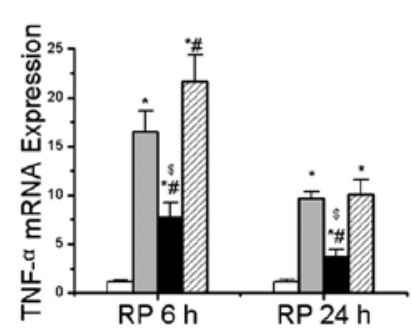

$\mathrm{L}$

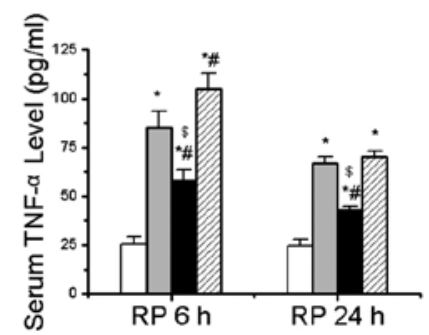

Figure 6. Alda-1 pretreatment inhibits the inflammatory response. (A) Western blotting was used to analyze the expression levels of HMGB1 and TLR4 in liver tissues after 6 and $24 \mathrm{~h}$ of reperfusion. Relative expression was normalized against the $\beta$-actin loading control. Densitometric semi-quantification of (B) HMGB1 and (C) TLR4 protein expression levels from part. (A) mRNA expression levels of (D) HMGB1, (E) TLR4, (F) IFN- $\gamma$, (G) IL-1 $\beta$, (H) IL-6 and (I) TNF- $\alpha$ after 6 and $24 \mathrm{~h}$ of reperfusion. Serum levels of (J) IL-1 $\beta$, (K) IL- 6 and (L) TNF- $\alpha$ after 6 and 24 h of reperfusion. Data are presented as the mean \pm SD. $n=6 .{ }^{*} \mathrm{P}<0.05$ vs. the sham group; ${ }^{*} \mathrm{P}<0.05$ vs. the IR group; ${ }^{~} \mathrm{P}<0.05$ vs. the IR-Daidzin group. $\mathrm{RP}$, reperfusion; HMGB1, high mobility group box 1 ; TLR4, toll-like receptor 4; IFN- $\gamma$, interferon- $\gamma$; IL, interleukin; TNF- $\alpha$, tumor necrosis factor- $\alpha$; IR, ischemia/reperfusion.

results strongly suggested that Alda-1 pretreatment may significantly alleviate HIRI by inducing autophagy.

Alda-1 pretreatment-induced autophagy in HIRI is mediated by AKT/mTOR and AMPK signaling activation. To assess the potential regulatory factors underlying autophagy induction, the activity levels of the AKT/mTOR and AMPK signaling pathways were analyzed. Compared with the sham group, the IR group had significantly downregulated phosphorylation levels of AKT at Thr308 and of AMPK at Thr172, while upregulated expression levels of p-mTOR at Ser2448 and p-S6 at Ser240/244 were observed (Fig. 9A-E). These effects were significantly reversed in the IR-Alda-1 group. Thus, these findings suggested that ALDH2-induced autophagy in HIRI may be dependent on the AKT/mTOR and AMPK signaling pathways.

\section{Discussion}

HIRI is a complex pathophysiological process that often leads to poor clinical prognosis. Clinically, there are no effective means to alleviate HIRI. The aim of the present study was to evaluate the effects of Alda-1 on HIRI and to examine the underlying mechanisms. In the present study, the pretreatment with Alda-1, an ALDH2 activator, reduced the negative effects of HIRI, including hepatic enzyme injury, 4-HNE levels, oxidative stress, hepatocyte apoptosis and inflammation. The observed decrease in HIRI-induced hepatic necrosis, oxidative stress and inflammatory responses were revealed to be associated with the inhibition of autophagy. In addition, Alda-1 was discovered to protect the liver following HIRI by enhancing autophagy and restoring the autophagy flux. It was also identified that the Alda-1-induced autophagy 


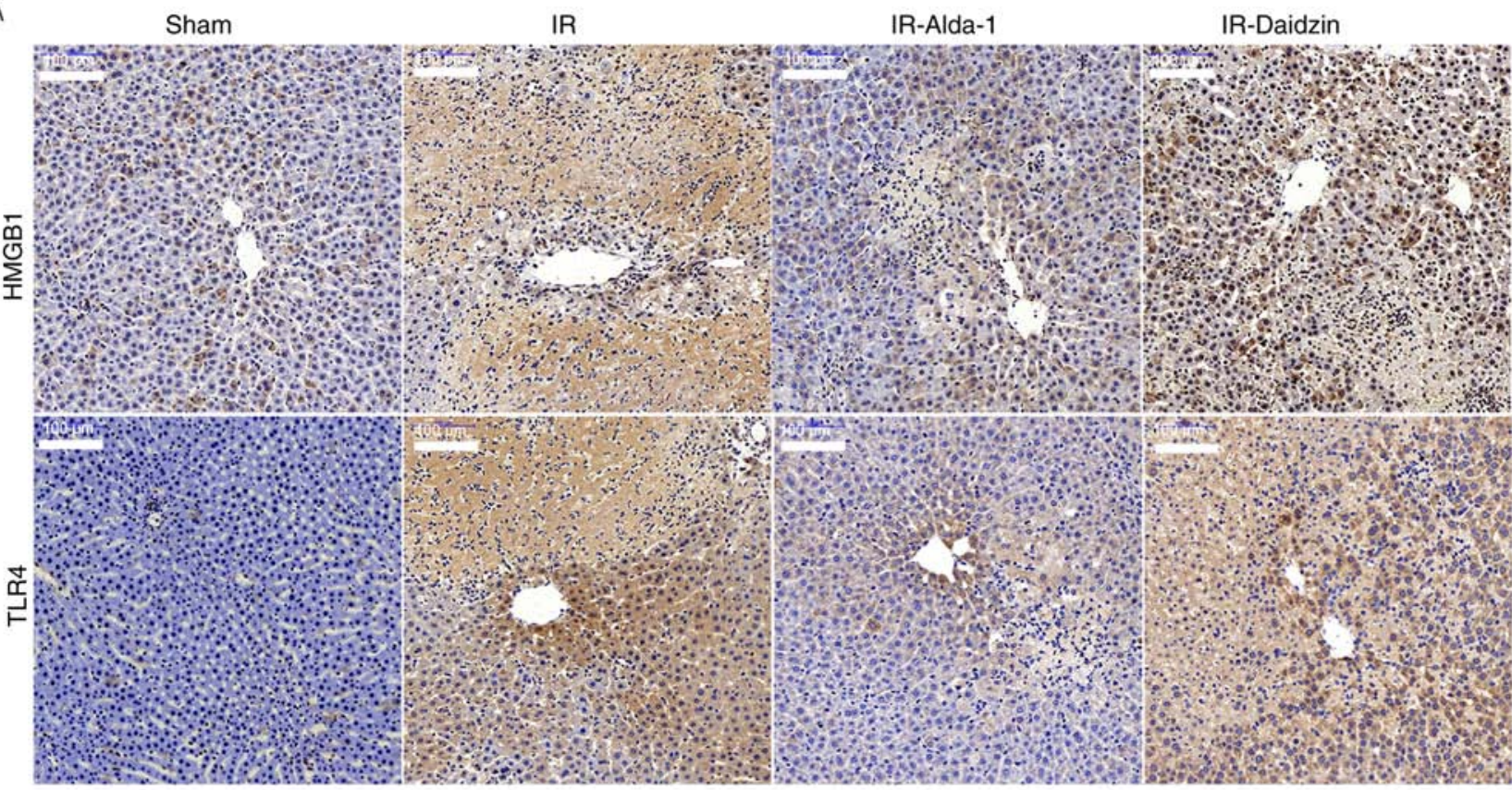

B
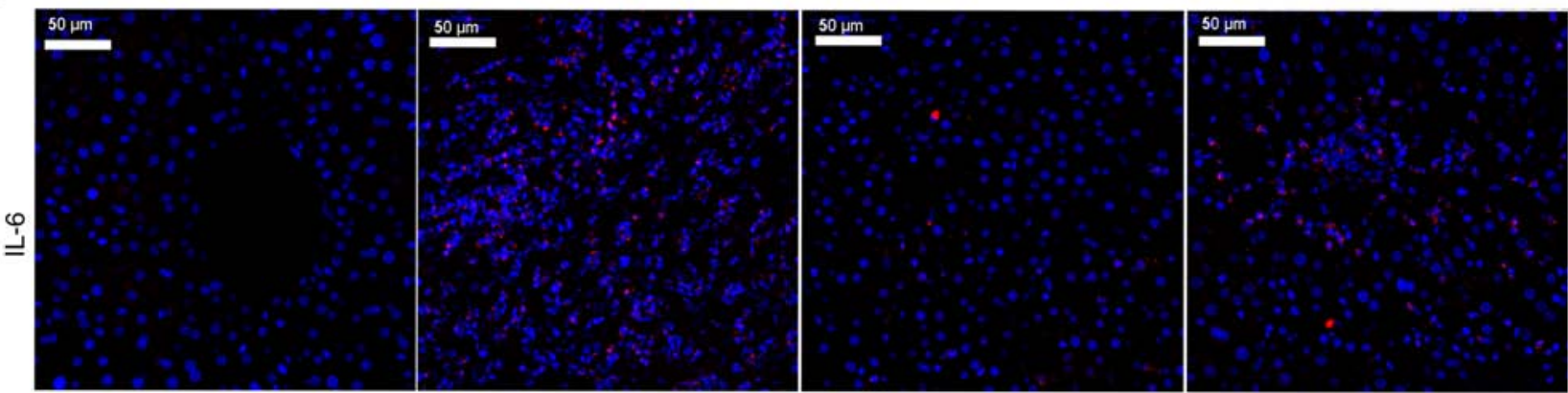

Figure 7. Alda-1 pretreatment reduces the distribution of HMGB1, TLR4 and release of IL-6. (A) Immunohistochemical staining was used to determine the expression levels of HMGB1 and TLR4 after $24 \mathrm{~h}$ of reperfusion. Magnification, x 200; scale bar, $100 \mu \mathrm{m}$. (B) Immunofluorescence images showing IL-6 expression levels after $24 \mathrm{~h}$ of reperfusion. Magnification, $\mathrm{x} 400$; scale bar, $50 \mu \mathrm{m}$. $\mathrm{n}=6$. HMGB1, high mobility group box 1; TLR4, toll-like receptor 4; IL, interleukin; IR, ischemia/reperfusion.

may be dependent on the AKT/mTOR and AMPK signaling pathways.

Previous studies have suggested that Alda-1 had a protective effect on myocardial IRI (5) and liver injuries caused by chronic alcohol intake $(12,21)$. However, the mechanisms underlying the effects of Alda-1 pretreatment on HIRI remain poorly understood. To address this knowledge gap, a rat model of $70 \%$ HIRI was established to investigate whether Alda-1 pretreatment could reduce the negative effects of HIRI and to characterize the underlying mechanisms. Warm ischemia caused liver function damage after 6 and $24 \mathrm{~h}$ of reperfusion. The pretreatment with Alda-1 led to a significant reduction in the serum levels of ALT and AST. Extensive hepatocyte necrosis, the congestion of the blood cells in the liver sinusoid and neutrophil infiltration all were identified in H\&E-stained histological sections from the IR group after 6 and 24 h of reperfusion. These effects were markedly reduced in the group pretreated with Alda-1. Thus, Alda-1 pretreatment was suggested to exert a strong protective effect against HIRI in the rat model. However, the mechanism through which Alda-1 protects the liver against HIRI remains to be clarified.
Previous studies have reported that Alda-1 exerts beneficial effects on ischemia injury in other organ models, including the intestine (22), kidney (23) and brain (24). Changes in ALDH2 expression levels were evaluated in HIRI rats; however, in the present study, it was discovered that Alda-1 did not affect the ALDH2 protein expression levels. Nonetheless, HIRI decreased ALDH2 activity. More importantly, the ALDH2 activity in HIRI rats was improved by Alda-1 pretreatment.

Reperfusion injury has been reported to results in the intracellular and extracellular release of ROS, which may be generated by mitochondrial dysfunction $(3,25)$. Furthermore, reperfusion injury was discovered to promote the accumulation of toxic reactive aldehydes, such as MDA and 4-HNE $(26,27)$. Doorn et al (11) identified that 4-HNE accumulation was increased by myocardial ischemia injury reperfusion in mice, and that this increase was reversed by ALDH2. In addition, ROS, MDA and 4-HNE have all been identified as important markers of oxidative stress in HIRI $(4,8,9,28)$, and oxidative stress is associated with all stages of HIRI (28). Thus, strategies aimed at reducing oxidative stress may also improve HIRI. In the present 
A

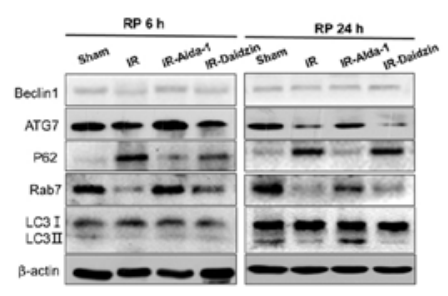

D

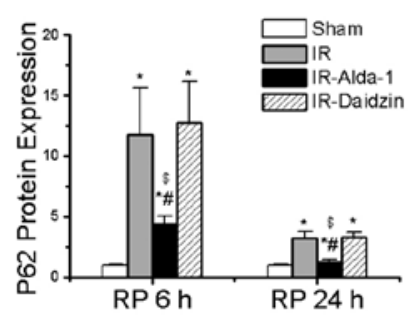

B

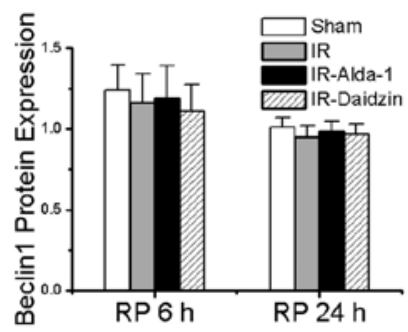

$\mathrm{E}$

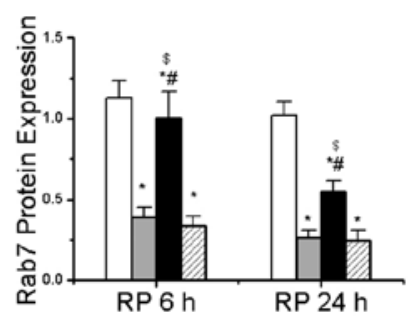

C

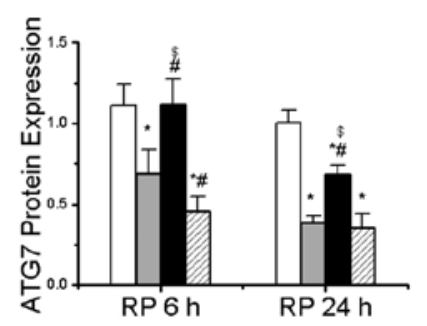

F

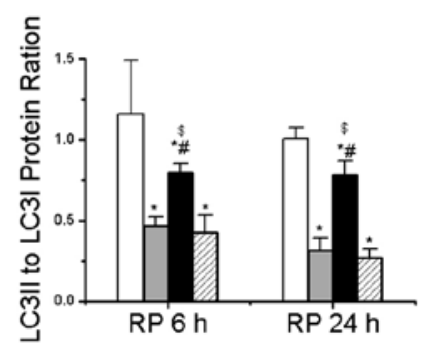

Figure 8. Alda-1 pretreatment reverses the hepatic IR injury-induced suppression of autophagy. (A) Western blotting was used to analyze the expression levels of Beclin1, ATG7, p62, Rab7 and LC3 in the liver tissues after 6 and $24 \mathrm{~h}$ of reperfusion. Relative expression was normalized against the $\beta$-actin loading control. Densitometric semi-quantification of the expression levels of (B) Beclin1, (C) ATG7, (D) p62, (E) Rab7 and (F) LC3 ratio from part. (A) Data are presented as the mean \pm SD. $n=6$. ${ }^{*} \mathrm{P}<0.05$ vs. the sham group; ${ }^{*} \mathrm{P}<0.05$ vs. the IR group; ${ }^{\$} \mathrm{P}<0.05$ vs. the IR-Daidzin group. ATG7, autophagy-related 7 ; LC3, microtubule associated protein 1 light chain $3 \alpha$; IR, ischemia/reperfusion; RP, reperfusion.

A

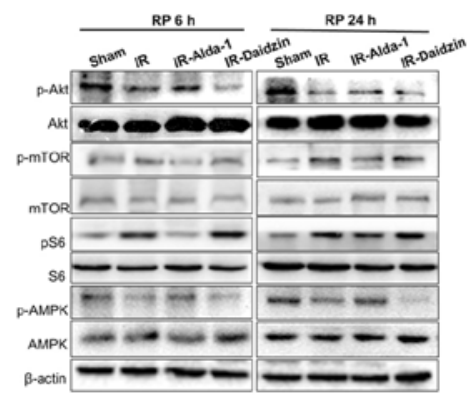

C

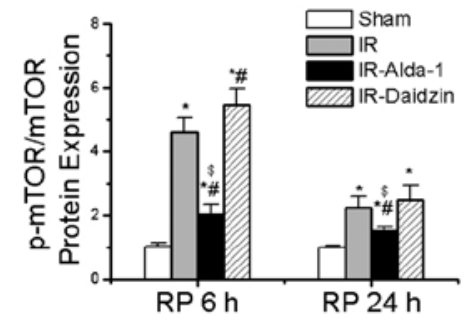

B

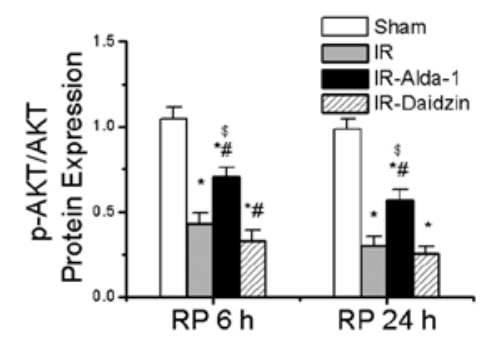

$\mathrm{E}$

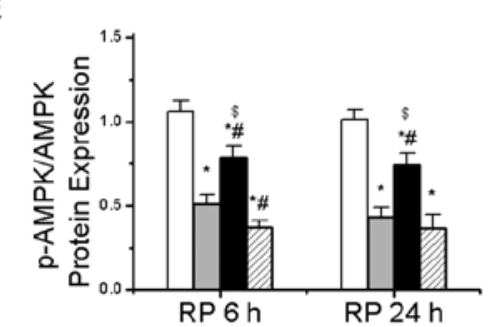

Figure 9. Alda-1 pretreatment enhances autophagy in rats with hepatic IR injury through activation of AKT/mTOR and AMPK signaling. (A) Western blotting was used to analyze the expression levels of AKT, p-AKT at Thr308, mTOR, p-mTOR at Ser2448, S6, p-S6 at Ser240/244, AMPK and p-AMPK at Thr172 in liver tissues after 6 and $24 \mathrm{~h}$ of reperfusion. Relative expression was normalized against the $\beta$-actin loading control. Densitometric semi-quantification of (B) the ratio of p-AKT protein expression to total AKT protein expression, (C) the ratio of p-mTOR protein expression to total mTOR protein expression, (D) the ratio of p-S6 protein expression to total S6 protein expression and (E) p-AMPK protein expression to total AMPK protein expression from part. (A) Data are presented as the mean $\pm \mathrm{SD}$. $\mathrm{n}=6$. ${ }^{\mathrm{P}} \mathrm{P}<0.05$ vs. the sham group; ${ }^{*} \mathrm{P}<0.05$ vs. the IR group; ${ }^{\mathrm{S}} \mathrm{P}<0.05$ vs. the IR-Daidzin group. mTOR, mammalian target of rapamycin; AMPK, AMP-activated protein kinase $\alpha$; p, phosphorylated; IR, ischemia/reperfusion; RP, reperfusion.

study, consistent with previous studies, HIRI induced mitochondrial damage, including an increase in 4-HNE, ROS and MDA production. Restoration of ALDH2 activity eliminated the accumulation of 4-HNE, ROS and MDA. Traverso et al (29) suggested that the formation of the 4-HNE adduct decreased ALDH2 activity. In addition, during reperfusion injury, mitochondrial dysfunction and oxidative stress were identified to contribute to cell death (4). In the present study, TUNEL staining of the liver sections indicated that fewer apoptosis-positive cells were present following Alda-1 pretreatment compared with the IR group. These findings suggested that Alda-1 pretreatment may improve HIRI by reducing oxidative stress, mitochondrial injury and hepatocyte apoptosis. 
Damage-associated molecular pattern (DAMP) signaling has been found to serve a critical role in HIRI pathogenesis $(4,30)$. DAMPs are produced by apoptotic and necrotic hepatocytes (4). At the start of reperfusion, DAMPs have been observed to rapidly activate the innate immune system and initiate an inflammatory response (4). Moreover, the reintroduction of blood was found to exacerbate ROS generation, leading to necrotic liver cell death (31), further increasing DAMP release (4). The increased release of endogenous DAMPs was discovered to be detrimental to organ function, negatively affecting HMGB1 and TLRs activity (32). Furthermore, the release of hepatocyte HMGB1 has been identified to critically depend on intracellular ROS accumulation (33) and HMGB1 was discovered to be scavenged by TLR4 (34). In the present study, it was hypothesized that Alda-1 pretreatment may protect liver tissue against HIRI by inhibiting inflammatory responses. The expression levels of HMGB1, TLR4, IFN- $\gamma$, IL-1 $\beta$, IL- 6 and TNF- $\alpha$ were significantly increased in the IR group. However, ALDH2 activation by Alda-1 pretreatment inhibited the expression levels of these proteins, indicating that Alda-1 may improve HIRI by inhibiting the inflammatory response.

Autophagy is a dynamic, intracellular, degradative process that targets damaged organelles, dysfunctional proteins and harmful products (20). Autophagy is induced under hypoxia and ischemia (35), and involves the formation of autolysosomes and autophagosomes (20). Several previous studies indicated that autophagy could protect the liver from HIRI, and that the inhibition of autophagy in HIRI could increase cell death (36-38). Kim et al (39) demonstrated that HIRI impaired autophagy in mice, which was associated with a marked decrease in the expression levels of LC3, ATG7 and Beclin1. This finding suggested that the enhancement of autophagy might represent a novel treatment option for HIRI. Moreover, Wang et al (38) demonstrated that increasing autophagy ameliorated liver damage and improved mitochondrial function against HIRI in aged mice. Liu et al (40) also suggested that ischemic pre-conditioning ameliorated HIRI in rats by inducing autophagy. Altogether, these previous studies strongly suggested that autophagy may be a protective mechanism against HIRI.

Guo et al (12) demonstrated that ALDH2 ameliorated hepatic damage due to chronic alcohol intake via the induction of autophagy. However, the potential mechanisms underlying the association between ALDH2 and autophagy in HIRI remain uncertain. In the present study, Alda-1 pretreatment increased autophagy in HIRI. Thus, it could be suggested that Alda-1 pretreatment may protect the liver via the induction of autophagy.

Further studies are required to identify the mechanisms regulating autophagy during Alda-1 pretreatment of HIRI. Zhang et al (41) demonstrated that activated p-AKT significantly attenuated HIRI. In addition, activated p-AKT has been demonstrated to serve a pivotal role in autophagy (42). In cardiomyocyte IRI, glucose deprivation induced autophagy through the inhibition of p-mTOR and the activation of p-AMPK (43). Interestingly, p-mTOR was reported to be negatively regulated by p-AKT (44). Ma et al (6) demonstrated that $\mathrm{ALDH} 2$ rescued myocardial IRI by regulating autophagy via AKT/mTOR and AMPK signaling. In the present study, Alda-1 pretreatment upregulated the expression levels of p-AKT and p-AMPK and downregulated the expression levels of $\mathrm{p}$-mTOR. Thus, ALDH2-induced autophagy might be dependent on the AKT/mTOR and AMPK signaling pathways, and these pathways may serve a role in the observed protective effects of Alda-1 pretreatment on HIRI.

However, the present study had two experimental limitations. The study design was based on animal experiments in vivo, and the results were not verified in vitro. In addition, the role of ALDH2 in conferring protection against HIRI was not confirmed using ALDH2 overexpression or knockdown tools.

In conclusion, Alda-1, an ALDH2 activator, was discovered to alleviate HIRI-induced effects, including hepatic enzyme injuries, acetaldehyde accumulation, oxidative stress, hepatocyte apoptosis and inflammation, possibly through the induction of autophagy. Thus, ALDH2 may be a potential target of new pharmacological strategies applicable to HIRI clinical practice.

\section{Acknowledgements}

Not applicable.

\section{Funding}

The present study was supported by the Medical Science Advancement Program (Clinical Medicine) of Wuhan University (grant no. TFLC 2018003) and the Program of Excellent Doctoral (Postdoctoral) of Zhongnan Hospital of Wuhan University (grant no. ZNYB2019008).

\section{Availability of data and materials}

The datasets used and/or analyzed during the current study are available from the corresponding author on reasonable request.

\section{Authors' contributions}

ZL and QY designed and performed the research, analyzed the data and wrote the article. XF, LL, YX and ZZ performed the experiments, contributed ideas and helped write the article. ZL, XZ, PY, CL, JL, XH, WY and WW helped establish the in vivo model. YW provided guidance and revised the article. YW, QY and SY designed the experiments, provided overall guidance and helped with the manuscript. All authors read and approved the final manuscript.

\section{Ethics approval and consent to participate}

All animal experiments and protocols were approved by The Committee on the Experimental Animal Regulations of The Zhongnan Hospital of Wuhan University (approval no. A237; Wuhan, China) and conformed to The Guide for the Care and Use of Laboratory Animals (15).

\section{Patient consent for publication}

Not applicable. 


\section{Competing interests}

The authors declare that they have no competing interests.

\section{References}

1. Selzner N, Rudiger H, Graf R and Clavien PA: Protective strategies against ischemic injury of the liver. Gastroenterology 125 917-936, 2003

2. Uchida Y, Ke B, Freitas MC, Yagita H, Akiba H, Busuttil RW, Najafian $\mathrm{N}$ and Kupiec-Weglinski JW: T-cell immunoglobulin mucin-3 determines severity of liver ischemia/reperfusion injury in mice in a TLR4-dependent manner. Gastroenterology 139 2195-2206, 2010.

3. Peralta C, Jiménez-Castro MB and Gracia-Sancho J: Hepatic ischemia and reperfusion injury: Effects on the liver sinusoidal milieu. J Hepatol 59: 1094-1106, 2013.

4. van Golen RF, van Gulik TM and Heger M: The sterile immune response during hepatic ischemia/reperfusion. Cytokine Growth Factor Rev 23: 69-84, 2012.

5. Gomes KM, Bechara LR, Lima VM, Ribeiro MA, Campos JC Dourado PM, Kowaltowski AJ, Mochly-Rosen D and Ferreira JC: Aldehydic load and aldehyde dehydrogenase 2 profile during the progression of post-myocardial infarction cardiomyopathy: Benefits of Alda-1. Int J Cardiol 179: 129-138, 2015.

6. Ma H, Guo R, Yu L, Zhang Y and Ren J: Aldehyde dehydrogenase 2 (ALDH2) rescues myocardial ischaemia/reperfusion injury: Role of autophagy paradox and toxic aldehyde. Eur Heart J 32: 1025-1038, 2011

7. Chen CH, Ferreira JC, Gross ER and Mochly-Rosen D: Targeting aldehyde dehydrogenase 2: New therapeutic opportunities. Physiol Rev 94: 1-34, 2014.

8. Chen CH, Sun L and Mochly-Rosen D: Mitochondrial aldehyde dehydrogenase and cardiac diseases. Cardiovasc Res 88: 51-57, 2010.

9. Yoval-Sánchez B and Rodríguez-Zavala JS: Differences in susceptibility to inactivation of human aldehyde dehydrogenases by lipid peroxidation byproducts. Chem Res Toxicol 25: 722-729, 2012.

10. Gomes KM, Campos JC, Bechara LR, Queliconi B, Lima VM, Disatnik MH, Magno P, Chen CH, Brum PC, Kowaltowski AJ, et al: Aldehyde dehydrogenase 2 activation in heart failure restores mitochondrial function and improves ventricular function and remodelling. Cardiovasc Res 103: 498-508, 2014.

11. Doorn JA, Hurley TD and Petersen DR: Inhibition of human mitochondrial aldehyde dehydrogenase by 4-hydroxynon-2-enal and 4-oxonon-2-enal. Chem Res Toxicol 19: 102-110, 2006.

12. Guo R, Xu X, Babcock SA, Zhang Y and Ren J: Aldehyde dedydrogenase-2 plays a beneficial role in ameliorating chronic alcohol-induced hepatic steatosis and inflammation through regulation of autophagy. J Hepatol 62: 647-656, 2015.

13. Chen CH, Budas GR, Churchill EN, Disatnik MH, Hurley TD and Mochly-Rosen D: Activation of aldehyde dehydrogenase-2 reduces ischemic damage to the heart. Science 321: 1493-1495, 2008

14. Czaja MJ, Ding WX, Donohue TJ Jr, Friedman SL, Kim JS, Komatsu M, Lemasters JJ, Lemoine A, Lin JD, Ou JH, et al: Functions of autophagy in normal and diseased liver. Autophagy 9: 1131-1158, 2013.

15. National Research Council: Guide for the care and use of laboratory animals. 8th edition. The National Academies Press, Washington, DC, 2011.

16. Ji W, Wei S, Hao P, Xing J, Yuan Q, Wang J, Xu F and Chen Y: Aldehyde dehydrogenase 2 has cardioprotective effects on myocardial ischaemia/reperfusion injury via suppressing mitophagy. Front Pharmacol 7: 101, 2016.

17. Suzuki S, Toledo-Pereyra LH, Rodriguez FJ and Cejalvo D: Neutrophil infiltration as an important factor in liver ischemia and reperfusion injury. Modulating effects of FK506 and cyclosporine. Transplantation 55: 1265-1272, 1993.

18. Liu Z, Zhang X, Xiao Q, Ye S, Lai CH, Luo J, Huang X, Wang W, Zeng C, Zhong Z, et al: Pretreatment donors after circulatory death with simvastatin alleviates liver ischemia reperfusion injury through a KLF2-dependent mechanism in rat. Oxid Med Cell Longev 2017: 3861914, 2017.
19. Livak KJ and Schmittgen TD: Analysis of relative gene expression data using real-time quantitative PCR and the 2(-Delta Delta $\mathrm{C}(\mathrm{T})$ ) method. Methods 25: 402-408, 2001

20. Cursio R, Colosetti P and Gugenheim J: Autophagy and liver ischemia-reperfusion injury. Biomed Res Int 2015: 417590, 2015.

21. Zhong W, Zhang W, Li Q, Xie G, Sun Q, Sun X, Tan X, Sun X, Jia W and Zhou Z: Pharmacological activation of aldehyde dehydrogenase 2 by Alda- 1 reverses alcohol-induced hepatic steatosis and cell death in mice. J Hepatol 62: 1375-1381, 2015.

22. Zhu Q, He G, Wang J, Wang Y and Chen W: Pre-treatment with the ALDH2 agonist Alda-1 reduces intestinal injury induced by ischaemia and reperfusion in mice. Clin Sci (Lond) 131: 1123-1136, 2017

23. Zhong Z, Hu Q, Fu Z, Wang R, Xiong Y, Zhang Y, Liu Z, Wang Y and Ye Q: Increased expression of aldehyde dehydrogenase 2 reduces renal cell apoptosis during ischemia/reperfusion injury after hypothermic machine perfusion. Artif Organs 40: 596-603, 2016.

24. Fu SH, Zhang HF, Yang ZB, Li TB, Liu B, Lou Z, Ma QL, Luo XJ and Peng J: Alda-1 reduces cerebral ischemia/reperfusion injury in rat through clearance of reactive aldehydes. Naunyn Schmiedebergs Arch Pharmacol 387: 87-94, 2014.

25. Caraceni P, Domenicali M, Vendemiale G, Grattagliano I, Pertosa A, Nardo B, Morselli-Labate AM, Trevisani F, Palasciano G, Altomare E and Bernardi M: The reduced tolerance of rat fatty liver to ischemia reperfusion is associated with mitochondrial oxidative injury. J Surg Res 124: 160-168, 2005.

26. Horton JW and Walker PB: Oxygen radicals, lipid peroxidation, and permeability changes after intestinal ischemia and reperfusion. J Appl Physiol (1985) 74: 1515-1520, 1993.

27. Lee H, Ko EH, Lai M, Wei N, Balroop J, Kashem Z and Zhang M: Delineating the relationships among the formation of reactive oxygen species, cell membrane instability and innate autoimmunity in intestinal reperfusion injury. Mol Immunol 58: 151-159, 2014.

28. van Golen RF, van Gulik TM and Heger M: Mechanistic overview of reactive species-induced degradation of the endothelial glycocalyx during hepatic ischemia/reperfusion injury. Free Radic Biol Med 52: 1382-1402, 2012.

29. Traverso N, Menini S, Odetti P, Pronzato MA, Cottalasso D and Marinari UM: Diabetes impairs the enzymatic disposal of 4-hydroxynonenal in rat liver. Free Radic Biol Med 32: 350-359, 2002.

30. Zhang Y, Yuan D, Yao W, Zhu Q, Liu Y, Huang F, Feng J, Chen X, Huang Y, Chi X and Hei Z: Hyperglycemia aggravates hepatic ischemia reperfusion injury by inducing chronic oxidative stress and inflammation. Oxid Med Cell Longev 2016: 3919627, 2016

31. Bhogal RH, Curbishley SM, Weston CJ, Adams DH and Afford SC: Reactive oxygen species mediate human hepatocyte injury during hypoxia/reoxygenation. Liver Transpl 16: 1303-1313, 2010.

32. Gracia-Sancho J, Villarreal GJ Jr, Zhang Y, Yu JX, Liu Y, Tullius SG and García-Cardeña G: Flow cessation triggers endothelial dysfunction during organ cold storage conditions: Strategies for pharmacologic intervention. Transplantation 90: 142-149, 2010.

33. Tsung A, Klune JR, Zhang X, Jeyabalan G, Cao Z, Peng X, Stolz DB, Geller DA, Rosengart MR and Billiar TR: HMGB1 release induced by liver ischemia involves Toll-like receptor 4 dependent reactive oxygen species production and calcium-mediated signaling. J Exp Med 204: 2913-2923, 2007.

34. Tsung A, Sahai R, Tanaka H, Nakao A, Fink MP, Lotze MT, Yang H, Li J, Tracey KJ, Geller DA and Billiar TR: The nuclear factor HMGB1 mediates hepatic injury after murine liver ischemia-reperfusion. J Exp Med 201: 1135-1143, 2005.

35. Dong Y, Undyala VV, Gottlieb RA, Mentzer RJ Jr and Przyklenk K: Autophagy: Definition, molecular machinery, and potential role in myocardial ischemia-reperfusion injury. J Cardiovasc Pharmacol Ther 15: 220-230, 2010.

36. Liu A, Fang H, Dahmen U and Dirsch O: Chronic lithium treatment protects against liver ischemia/reperfusion injury in rats. Liver Transpl 19: 762-772, 2013.

37. Wang JH, Ahn IS, Fischer TD, Byeon JI, Dunn WA Jr, Behrns KE, Leeuwenburgh C and Kim JS: Autophagy suppresses age-dependent ischemia and reperfusion injury in livers of mice. Gastroenterology 141: 2188-2199, 2011.

38. Wang JH, Behrns KE, Leeuwenburgh C and Kim JS: Critical role of autophage in ischemia/reperfusion injury to aged livers. Autophagy 8: 140-141, 2012. 
39. Kim JS, Nitta T, Mohuczy D, O'Malley KA, Moldawer LL, Dunn WA Jr and Behrns KE: Impaired autophagy: A mechanism of mitochondrial dysfunction in anoxic rat hepatocytes. Hepatology 47: 1725-1736, 2008.

40. Liu A, Fang H, Wei W, Dirsch O and Dahmen U: Ischemic preconditioning protects against liver ischemia/reperfusion injury via heme oxygenase-1-mediated autophagy. Crit Care Med 42: e762-e771, 2014.

41. Zhang R, Zhang L, Manaenko A, Ye Z, Liu W and Sun X: Helium preconditioning protects mouse liver against ischemia and reperfusion injury through the PI3K/Akt pathway. J Hepatol 61: 1048-1055, 2014.

42. Arico S, Petiot A, Bauvy C, Dubbelhuis PF, Meijer AJ, Codogno P and Ogier-Denis E: The tumor suppressor PTEN positively regulates macroautophagy by inhibiting the phosphatidylinositol 3-kinase/protein kinase B pathway. J Biol Chem 276: 35243-35246, 2001.
43. Matsui Y, Takagi H, Qu X, Abdellatif M, Sakoda H, Asano T,Levine B and Sadoshima J: Distinct roles of autophagy in the heart during ischemia and reperfusion: Roles of AMP-activated protein kinase and Beclin 1 in mediating autophagy. Circ Res 100: 914-922, 2007.

44. Inoki K, Li Y, Zhu T, Wu J and Guan KL: TSC2 is phosphorylated and inhibited by Akt and suppresses mTOR signalling. Nat Cell Biol 4: 648-657, 2002.

(i) (2) This work is licensed under a Creative Commons Attribution-NonCommercial-NoDerivatives 4.0 International (CC BY-NC-ND 4.0) License. 